\title{
IL-13 and adenosine: partners in a molecular dance?
}

\author{
Gabriele Grünig
}

Department of Pathology, J.P. Mara Center for Lung Research, Columbia University and St. Luke's Roosevelt Hospital, New York, New York, USA

\begin{abstract}
Inflammation and airway remodeling are two responses readily apparent in asthma and other inflammatory disorders of the airway and lungs. Both adenosine and IL-13 play critical roles in contributing pathways. A new study (see the related article beginning on page 332) reveals a previously unrecognized interaction between adenosine and IL-13 that indicates a mutual stimulation that may contribute to the nature and severity of airway inflammation and fibrosis.
\end{abstract}

J. Clin. Invest. 112:329-331 (2003). doi:10.1172/JCI200319392.

Asthma has puzzled medical scientists for centuries. Its complexity is due to the multitude of pathological changes that affect diverse cell types and the multitude of pathogenetic pathways that induce these changes. Altered smooth-muscle responses result in increased airway resistance and increased responses to nonspecific stimuli, a phenomenon known as airway hyperreactivity. Interactions between eosinophils, mast cells, $\mathrm{T}$ cells, B cells, and IgE antibodies result in airway inflammation. Altered airway epithelial cell and fibroblast maturation cause airway remodeling, which is characterized by basement membrane fibrosis and increased mucus production. In this issue of the JCI, Blackburn and colleagues (1) show that the molecular cascades initiated by adenosine or IL-13 signaling are interconnected. Mice that express an IL-13 transgene in the airways (IL-13-transgenic mice) had increased adenosine levels and reduced adensosine deaminase (ADA) gene expres-

Address correspondence to: Gabriele Grünig, St. Luke's Roosevelt Hospital, 432 West 58th Street, Laboratory 501, New York, New York 10019, USA.

Phone: (212) 523-4765; Fax: (212) 523-8005; E-mail: gg398@columbia.edu.

Conflict of interest: The author has declared that no conflict of interest exists.

Nonstandard abbreviations used: adenosine deaminase (ADA); severe combined immunodeficiency (SCID); chronic obstructive pulmonary disease (COPD); IL-13 receptor $\alpha 1$ (IL-13R $\alpha 1$ ); signal transducer and activator of transcription-6 (STAT6). sion and ADA enzyme activity in the lungs. Furthermore, the expression of the proinflammatory adenosine receptors $A_{1}, A_{2 B}$, and $A_{3}$ was significantly increased in the lungs of IL-13transgenic mice while the expression of the anti-inflammatory $\mathrm{A}_{2 \mathrm{~A}}$ receptor was decreased. Furthermore, ADA treatment of IL-13-transgenic mice was shown to significantly reduce the level of bronchoalveolar lavage eosinophils and lung collagen content while mucus expression by epithelial cells or parenchymal inflammation was much less affected. Conversely, the authors showed that IL-13 levels were significantly increased in the inflamed lungs of ADA-deficient mice. Blackburn et al. conclude that their findings demonstrate a positive feedback loop whereby IL-13 induces adenosine expression and, in turn, adenosine stimulates IL-13 production. The authors hypothesize that IL-13induced adenosine and adenosineinduced IL-13 production stimulate proinflammatory receptors or mediators that may play important roles in the pathogenesis of asthma and chronic obstructive pulmonary disease (COPD).

\section{Adenosine, IL-13, and lung injury}

Adenosine is well recognized for inducing bronchoconstriction in asthmatic humans (2). It has also been shown to mediate lung injury in a mouse model of ADA deficiency and severe combined immunodefi- ciency (SCID) (3). These mice have hyperreactive airways, goblet cell hyperplasia, and eosinophilic airway inflammation (3). A critical role for IL-13 in elaborating the asthma phenotype (e.g., goblet cell hyperplasia, airway eosinophilia, airway hyperreactivity, subepithelial fibrosis) in mice has been demonstrated by many authors (4-6). These studies have shown that the allergen-induced asthma phenotype could be blocked by IL-13 inhibitors and that the asthma phenotype was elaborated by exposing the airways of mice to recombinant IL-13 or to IL-13 expressed by a transgene in airway epithelial cells. Thus far, there is no direct evidence supporting a role for IL-13 in human asthma. However, indirect evidence has been reported: polymorphisms in the $I L-13$ gene have been associated with asthma $(7$, $8)$; and human resident lung cells have been shown to react to IL-13 (9).

\section{Adenosine signaling and tissue injury}

Adenosine is released by stressed and dying cells and also in response to metabolic changes (Figure 1a). Free adenosine is removed by phosphorylation with adenosine kinase, or breakdown by ADA. Adenosine binds to $G$ protein-coupled receptors present in virtually all organs (reviewed in refs. 10 and 11). Different adenosine receptors are coupled to different $\mathrm{G}$ proteins and preferentially expressed by specific cell types. In asthmatic subjects, adenosine-mediated signaling in mast cells is thought to be central to inducing bronchoconstriction, an effect that has been utilized by many authors to diagnose airway hyperreactivity $(2,11)$. One of the targets of theophylline, a therapeutic drug for the treatment of asthma and COPD are the adenosine receptors. Adenosine also has significant effects in organs other than the lungs (10-12). In the immune system, high, uncontrolled levels of adenosine metabolites (as seen in ADA deficiency) are toxic to lymphocytes (12) and it is via this mechanism that ADA deficiency causes SCID in both humans and mice. 
IL-13 signaling and tissue injury

Th2 cells are a major source of IL-13 (Figure 1b) following stimulation by different types of antigens, including allergens. IL-13 can also be elicited in an antigen-independent manner during tissue injury. This may involve IL-25 (13), which releases IL-13 from yet undefined non-T cells. Eosinophils, basophils, and mast cells also produce IL-13. IL-13 receptors, of which IL-4 receptor- $\alpha$ and IL-13 receptor $\alpha 1$ (IL-13R $\alpha 1$ ) are core components, are present on many different cell types (with the exception of $T$ cells and murine $B$ cells). The common- $\gamma$ chain has also been observed as a non-IL-13-binding part of this receptor. IL-13 binding to its receptor leads to phosphorylation of the signal transducer and activator of transcription-6 (STAT6), which translocates to the nucleus and affects the transcription of many genes including lipoxygenases. A recent study by Vergraftig and colleagues (14) has shown that lipoxygenase products, such as leukotrienes, are critical mediators of IL-13-induced lung injury. IL-13 signaling is also fine-tuned by IL-13R $\alpha 2$, described by many authors, for example, in ref. 15, as a high-affinity IL-13 decoy receptor.

\section{Hypothetical interactions between IL-13 and adenosine}

While Blackburn et al. (1) have shown that IL-13 and adenosine interact and that positive feedback loops exist, the precise nature of their interaction remains unknown. Injured and dying cells may release adenosine in IL-13-transgenic mice (1), however this observation may not be specific for IL-13 and may also occur in many other types of lung inflammation. An alternative mechanism that may result in increased adenosine levels may involve IL-13-mediated regulation of ADA or adenosine kinase activities. In the lungs of ADA-deficient mice (1) IL-13 is most probably produced by non- $T$ cells as these mice have defective $T$ cells. The increased adenosine levels in ADA-deficient mice may induce IL-13 directly by stimulating mast cells or indirectly through
IL-25 release. At the receptor level, interactions may occur via regulation of IL-13R $\alpha 2$ expression or G protein-signaling. Blackburn et al. (1) demonstrate that specific adenosine receptors are upregulated in IL-13 transgenic mice and it is also known that IL-13 dramatically alters the expression of diverse signaling molecules in resident lung cells (9). Interactions between IL-13 and adenosine may also include subtle regulatory loops. Both adenosine

a

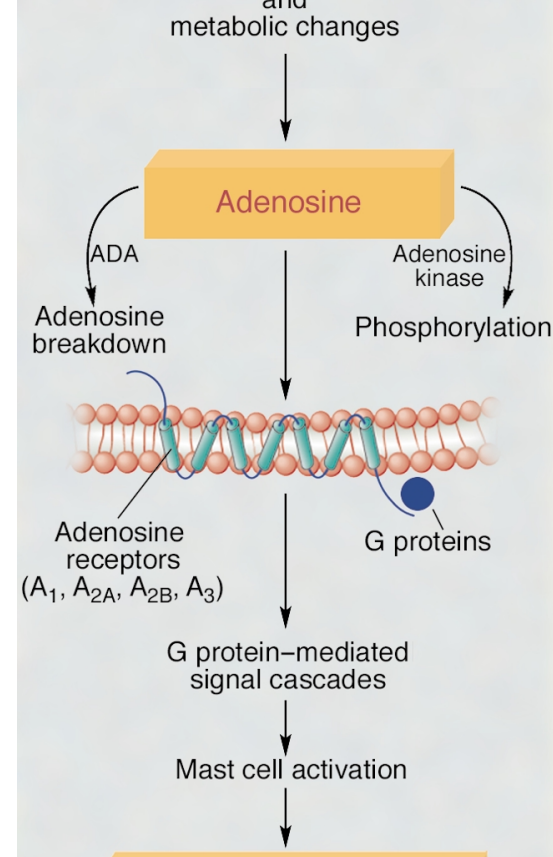

Airway hyperreactivity

Mucus overproduction

Eosinophilic inflammation and IL-13 affect enzymes that utilize arachidonic acid. Cyclooxygenases (11), the products of which may inhibit or enhance the asthma phenotype, are induced by adenosine. Lipoxygenases (14), which induce the asthma phenotype, are upregulated by IL-13. It must also be considered that negative feedback loops may occur: T cell inactivation mediated by increased levels of adenosine (12) may dampen the IL-13-initiated inflammatory program.

\section{Figure 1}

Adenosine and IL-13: sources, signaling, and effects in the lungs. (a) The adenosine-initiated cascade of molecular events is illustrated $(2,3,10,11)$. Overall adenosine levels reflect the balance between adenosine release by injured or metabolically stressed cells and adenosine metabolism by ADA or adenosine kinase. Adenosine receptors are $G$ protein-linked. This linkage provides a multitude of opportunities for the fine-tuning of the adenosine receptors. Mast cells are thought to be the major mediators of the adenosine-induced asthma phenotype. (b) The IL-13-initiated cascade of molecular events is illustrated (4-6, 13-15). IL-13 is expressed upon recognition of antigen (such as allergen), or in response to inflammatory stimuli (e.g., IL-25). IL-13 signaling results in the phosphorylation of the signal transducer and activator of transcription-6 (STAT6 $\left.{ }^{\mathrm{P}}\right)$ molecule and alters the expression of a multitude of genes in the lungs including the upregulation of the expression of lipoxygenase genes. Leukotrienes, the products of lipoxygenases, are thought to be important mediators of the IL-13-induced asthma phenotype. The precise molecular interactions between adenosine and IL-13 are not yet known. As shown by Blackburn et al. in this issue of the $J C I$ (1), positive feedback loops may involve IL-13-mediated regulation of ADA and adenosine receptors, and adenosine-mediated IL-13 release (1). A negative feedback loop may also exist. For example, toxic effects of high levels of adenosine on T cells (12) may dampen IL-13-mediated inflammation. 


\section{Conclusions}

The articles by Blackburn et al. (1) and by Vergraftig et al. (14) may signify a shift in the focus of asthma research away from the search for specific effector molecules and their relative importance, to the discovery of how known critical effector pathways interact. The work by Blackburn et al. (1) directs our focus towards exciting interactions between an old (adenosine) and a new (IL-13) effector molecule of asthma.

\section{Acknowledgments}

The author thanks Dean Sheppard and Gerard M. Turino for invaluable suggestions and advice.

1. Blackburn, M.R., et al. 2003. Adenosine mediates IL-13-induced inflammation and remodeling in the lung and interacts in an IL-13adenosine amplification pathway. J. Clin. Invest.
112:332-344. doi:10.1172/JCI200316815.

2. Van Den Berge, M., et al. 2001. PC(20) adenosine 5 -monophosphate is more closely associated with airway inflammation in asthma than PC(20) methacholine. Am. J. Respir. Crit. Care Med. 163:1546-1550.

3. Blackburn, M.R., et al. 2000. Metabolic consequences of adenosine deaminase deficiency in mice are associated with defects in alveogenesis, pulmonary inflammation, and airway obstruction. J. Exp. Med. 192:159-170.

4. Wills-Karp, M., et al. 1998. Interleukin-13: central mediator of allergic asthma. Science. 282:2258-2261.

5. Zhu, Z., et al. 1999. Pulmonary expression of interleukin-13 causes inflammation, mucus hypersecretion, subepithelial fibrosis, physiologic abnormalities, and eotaxin production. J. Clin. Invest. 103:779-788.

6. Grunig, G., et al. 1998. Requirement for IL-13 independently of IL-4 in experimental asthma. Science. 282:2261-2263.

7. Graves, P.E., et al. 2000. A cluster of seven tightly linked polymorphisms in the IL-13 gene is associated with total serum IgE levels in three populations of white children. J. Allergy Clin. Immunol. 105:506-513.

8. Howard, T.D., et al. 2002. Gene-gene interaction in asthma: IL4RA and IL13 in a Dutch population with asthma. Am. J. Hum. Genet. 70:230-236.
9. Lee, J.H., et al. 2001. Interleukin-13 induces dramatically different transcriptional programs in three human airway cell types. Am. J. Respir. Cell Mol. Biol. 25:474-485.

10. Linden, J. 2001. Molecular approach to adenosine receptors: receptor-mediated mechanism of tissue protection. Annu. Rev. Pharmacol. Toxicol. 41:775-787.

11. Fozard, J.R, and Hannon, J.P. 2000. Species differences in adenosine receptor-mediated bronchoconstrictor responses. Clin. Exp. Allergy 30:1213-1220.

12. Apasov, S.G., Blackburn, M.R., Kellems, R.E., Smith, P.T., and Sitkovsky, M.V. 2001. Adenosine deaminase deficiency increases thymic apoptosis and causes defective $\mathrm{T}$ cell receptor signaling. J. Clin. Invest. 108:131-141. doi:10.1172/JCI200110360.

13. Fort, M.M., et al. 2001. IL-25 induces IL-4, IL-5, and IL-13 and Th2-associated pathologies in vivo. Immunity. 15:985-995.

14. Vargaftig, B.B., and Singer, M. 2003. Leukotrienes mediate murine bronchopulmonary hyperreactivity, inflammation, and part of mucosal metaplasia and tissue injury induced by recombinant murine interleukin-13. Am. J. Respir. Cell Mol. Biol. 28:410-419.

15. Wood, N., et al. 2003. Enhanced interleukin (IL)-13 responses in mice lacking IL-13 receptor alpha 2. J. Exp. Med. 197:703-709. 